\title{
BMJ Open Prevalence and correlates of mental and neurodevelopmental symptoms and disorders among deaf children and adolescents: a systematic review protocol
}

\author{
Brandon Swanepoel (D) , ${ }^{1}$ Leslie Swartz (D) , ${ }^{1}$ Renate Gericke (D) , Sumaya Mall ${ }^{3}$
}

To cite: Swanepoel B, Swartz L, Gericke R, et al. Prevalence and correlates of mental and neurodevelopmental symptoms and disorders among deaf children and adolescents: a systematic review protocol. BMJ Open 2020;10:e038431. doi:10.1136/ bmjopen-2020-038431

- Prepublication history for this paper is available online. To view these files, please visit the journal online (http://dx.doi. org/10.1136/bmjopen-2020038431).

Received 16 March 2020 Revised 22 July 2020

Accepted 25 September 2020

D Check for updates

(c) Author(s) (or their employer(s)) 2020. Re-use permitted under CC BY-NC. No commercial re-use. See rights and permissions. Published by BMJ.

For numbered affiliations see end of article.

\section{Correspondence to} Mr Brandon Swanepoel; psychologistbrandon@gmail. com

\section{ABSTRACT}

Introduction Little is known of the prevalence and correlates of mental and neurodevelopmental symptoms and disorders among deaf children and adolescents. Research suggests that this is a vulnerable population group at high risk of these disorders. However, little is known of correlates of prevalence estimates of these mental disorders and it seems that heterogeneous tools have been used to derive these estimates. Given the heterogeneity of studies measuring the prevalence and correlates of mental and neurodevelopmental symptoms and disorders among deaf children and adolescents, we seek to systematically examine and synthesise observational epidemiological evidence in this area to articulate a more detailed account of these symptoms and disorders and their correlates among this population group.

Methods and analysis We will conduct a systematic search of the following electronic databases to identify published observational epidemiological studies examining the prevalence and correlates of mental and neurodevelopmental symptoms and disorders among deaf children and adolescents: EBSCOhost, ERIC, PsycARTICLES, PsycINF0, PubMED, ScienceDirect, SCOPUS and Web of Science. As research in this area is limited, eight databases have been included to widen our search to include as many articles as possible. The search terms will be related to mental and neurodevelopmental symptoms and disorders as well as deaf children and adolescents. Two reviewers will review and extract data from each article independently and, where relevant, discuss differences to reach consensus. Additionally, the reviewers will assess overall study quality and risk of bias using a quality appraisal scale. Findings from studies will be synthesised to produce a quantitative review that summarises existing evidence on mental and neurodevelopmental symptoms and disorders among deaf children and adolescents and their correlates. The publication date of studies will not be restricted so that as much data as possible that fit our inclusion criteria can be sourced. We will conduct our searches between August 2020 and March 2021.

Ethics and dissemination This systematic review will use publicly available data and therefore does not require a direct ethical review. The protocol was however
Strengths and limitations of this study

- To our knowledge, this is the first systematic review to synthesise rigorous prevalence and correlates of mental disorders in deaf children and adolescents.

- Inclusion criteria have been devised by a team of experienced researchers.

- Data synthesis and analysis will be based on a detailed assessment of methodological quality and risk of bias.

- If most of the studies are cross-sectional, we will have limited opportunity to infer causality or risk factors for the onset of mental disorders in deaf children and adolescents.

- We anticipate a paucity of research in the area and thus may have to include older studies.

submitted for ethics waiver clearance with Stellenbosch University Health Research Ethics Committee. The protocol will be disseminated in a peer-reviewed journal. The review protocol was registered with the PROSPERO International Prospective Register of systematic reviews (http://www.crd.york.ac.uk/PROSPER0).

PROSPERO registration number CRD42020189403.

\section{INTRODUCTION}

\section{Background}

Research suggests that deaf children and adolescents are at high risk of mental and neurodevelopmental disorders with prevalence figures ranging between $19 \%$ and $77 \%$. Despite the wide range these estimates present, they do suggest that the risk of mental disorders might be higher among deaf children and adolescents compared with hearing children and adolescents. ${ }^{1-9}$ Mental and neurodevelopmental disorders include intellectual disabilities, autism spectrum disorder, mood disorders, schizophrenia spectrum and psychotic disorders and trauma and stressrelated disorders, classified by the Diagnostic 
and Statistical Manual of Mental Disorders (DSM), the International Classification of Diseases (ICD) or similar manuals used in the study country.

Generalisation of these findings is however difficult, as studies seem to vary widely on the range of symptoms and disorders assessed, the instruments used to assess symptoms and disorders, sample characteristics and research participants. Some studies base results on questionnaires or checklists administered to parents and teachers ${ }^{24710}$ while others base findings on self-report questionnaires administered to adolescents. ${ }^{5}{ }^{11}$ It is worth noting that very few studies have based results on direct clinical assessments of deaf children and adolescents. Furthermore, sample characteristics in prevalence studies vary considerably in terms of aetiology of deafness, type and degree of hearing loss, age of hearingimpaired diagnosis, primary language, use of assistive device, educational level and any coexisting disabilities or comorbidities.

Although studies report a high prevalence of mental and neurodevelopmental symptoms and disorders among this group, very few studies investigate specific types of disorders that affect this group. Those that do, find deaf children and adolescents at risk of depression, anxiety, oppositional defiant disorder, conduct disorder, attention deficit hyperactivity disorder, psychosis, somatoform disorder and pain disorder. ${ }^{79}$

The causes of mental and neurodevelopmental disorders also remain unknown, as studies tend to focus on correlates that vary widely among studies. Correlates that have been reported include: communication and developmental delays, ${ }^{12}$ quality of parent-child communication, ${ }^{13}$ early detection of hearing loss ${ }^{14}$ degree of hearing loss, ${ }^{15} 16$ secondary disabilities, ${ }^{12} 17$ maternal stress, ${ }^{2}{ }^{18}$ physical and sexual abuse, ${ }^{19} 20$ teasing and bullying ${ }^{15}$ and type of school attended. ${ }^{21}$ To further understand the additional difficulties experienced by deaf children and adolescents, it is important to quantify and synthesise the findings to date.

Given the heterogeneity of studies measuring the prevalence and correlates of mental and neurodevelopmental symptoms and disorders among deaf children and adolescents, we seek to systematically examine and synthesise observational epidemiological evidence in this area. In doing so, we wish to examine and synthesise prevalence estimates and their correlates among this population group. To our knowledge, there are two published systematic reviews related to mental disorders in deaf children and adolescents: the first focus is on behaviour problems in deaf children and the interventions used to address these problems, ${ }^{22}$ and the second is limited to studies measuring emotional and behaviour problems among deaf children using one assessment tool, the Strengths and Difficulties Questionnaire (SDQ) ${ }^{23}$ Thus, while these reviews are valuable, their contribution is limited to an explication of behavioural problems in this population and their prevalence as assessed by the SDQ.

\section{Objectives}

The objective of this review is to systematically examine and synthesise observational epidemiological evidence of prevalence and correlates of mental and neurodevelopmental symptoms and disorders among deaf children and adolescents, thereby providing an in-depth examination of prevalence estimates and correlates among this population group.

\section{METHODS AND ANALYSIS Types of studies}

We summarise our inclusion and exclusion criteria in table 1. Our review will include English and non-English studies from high, middle and low-income countries. The specific inclusion criteria for this review include (1) peerreviewed, (2) observational, (3) cross-sectional and (4) cohort studies that (5) investigate the prevalence and, where available, correlates of mental and neurodevelopmental symptoms and disorders among all subgroups of school-going deaf children and adolescents (typically 6-18 years of age) (6) using validated questionnaires or standardised psychiatric assessments administered to (7) parents, teachers, clinicians or children to assess mental health. The various instruments and informants used will be specified in our data extraction table and in the article. We are aware that in some countries, deaf individuals may not reach the level of their hearing peers and can attend school past the age of 18 . We will include participants older than 18 years of age in our study on condition that they are still attending school.

\section{Type of participants}

All subgroups of school-going deaf participants will be included. Subgroups include individuals with coexisting disabilities (developmental, physical or otherwise), congenital or postlingual hearing loss, mild to profound hearing loss, oral or sign language communication users, participants with and without cochlear implants or hearing aids and those attending mainstream or specialised schooling. The different subgroups will be specified in our data extraction table and discussed in the review.

\section{Types of variables to be measured \\ Exposure variables}

The exposure variables will be all the correlates of mental and neurodevelopmental symptoms and disorders mentioned in the existing literature, for example, communication and developmental delays, quality of parent-child communication, early detection of hearing loss, degree of hearing loss, maternal stress, secondary disabilities, physical and sexual abuse, teasing and bullying and sociodemographic factors.

\section{Outcome variables}

The outcome variables will be all mental and neurodevelopmental symptoms and disorders as classified and defined by the DSM (all revisions thereof), the ICD (all 
Table 1 Inclusion and exclusion criteria

\begin{tabular}{|c|c|c|}
\hline & Included & Excluded \\
\hline \multirow[t]{2}{*}{ Publication type } & English and non-English. & $\begin{array}{l}\text { Grey literature, unpublished articles, } \\
\text { opinion pieces, case and narrative } \\
\text { reports, publications that do not } \\
\text { have primary data and a clear } \\
\text { description of methods used. }\end{array}$ \\
\hline & Any date. & \\
\hline Study population & $\begin{array}{l}\text { All subgroups of school going deaf children and adolescents } \\
\text { (typically aged 6-18 years of age). Subgroups include } \\
\text { individuals with coexisting disabilities (developmental, physical } \\
\text { or otherwise), congenital or postlingual hearing loss, mild to } \\
\text { profound hearing loss, oral or sign language communication } \\
\text { users, participants with and without cochlear implants or hearing } \\
\text { aids, and those attending mainstream or specialised schooling. } \\
\text { We are aware that in some countries, deaf individuals may not } \\
\text { reach the level of their hearing peers and can attend school past } \\
\text { the age of eighteen. We will include participants older than } 18 \\
\text { years of age in our study on condition that they are still attending } \\
\text { school. } \\
\text { Studies conducted in high, middle and low-income countries. }\end{array}$ & Participants not attending school. \\
\hline Exposure variables & $\begin{array}{l}\text { The exposure variables will be all the correlates of mental } \\
\text { and neurodevelopmental symptoms and disorders mentioned } \\
\text { in the existing literature for example, communication and } \\
\text { developmental delays, quality of parent-child communication, } \\
\text { early detection of hearing loss, degree of hearing loss, maternal } \\
\text { stress, secondary disabilities, physical and sexual abuse, teasing } \\
\text { and bullying and sociodemographic factors. }\end{array}$ & \\
\hline
\end{tabular}

Instruments that have not been validated and assessments that are not standardised.

DSM, Diagnostic and Statistical Manual of Mental Disorders; ICD, International Classification of Diseases.

revisions thereof) or similar manuals used in the study country (and revisions thereof) and assessed using validated instruments or standardised assessments. In our analysis, we will distinguish between diagnostic outcomes according to DSM or other algorithms, and outcomes in terms of symptoms, which may or may not reach the threshold of diagnostic caseness. We anticipate that several studies will assess symptoms (by use, eg, of standard questionnaires) but will not have a formal assessment of diagnosis.

\section{Search methods for identification of studies}

We will conduct a systematic search of the following electronic databases EBSCOHost, ERIC, PsycARTICLES, PsycINFO, PubMED, ScienceDirect, SCOPUS and Web of Science. Eight databases have been included to widen our search and to include as many articles as possible. We have developed a search strategy that will be adapted to different search engines (see table 2). The search strategy will include both free text and Medical Subject Heading terms. Duplicate articles generated by the search engines will be removed. In addition to database search results, reference sections of the included journal articles will also be reviewed to identify any relevant articles that were missed by search engines. We will also use citation indices to follow-up on articles that cite earlier articles found through our search. Restrictions on the publication date of studies that fit our inclusion criteria have not been imposed as our objective is to glean as much evidence as possible on what we expect to be an under-researched field. We will conduct our searches between August 2020 and March 2021.

\section{Exclusion criteria}

This systematic review will exclude (1) grey literature, (2) unpublished articles, (3) opinion pieces, (4) case reports, (5) narrative reports, (6) qualitative studies, (7) casecontrol studies, (8) randomised controlled trials (RCTs) 
Concept A: Mental disorders and neurodevelopmental disorders

Within Concept A, terms used will include:
Concept B: deafness

Within Concept $B$, terms used will include:

("mental disord"” OR "mental illness" OR "emotional disord"” OR "neurodevelopmental disord"” OR "intellectual disab"” OR "mental handicap" OR "mental retardation" OR "cognitive impair" OR autism* OR aspergers OR "attention deficit disord*" OR "attention deficit hyperactivity disord*" OR ADD OR ADHD OR "learning disord"” OR "tic disord"” OR "tourette disord"” OR "psychotic disord" OR schizo* OR "dysregulated mood disord"” OR "mood disord"” OR "bipolar disord" OR "manic depressive disord"” OR "manic depression" OR "cyclothymic disord" OR "depressive disord" OR depression OR suicide OR self-harm OR self-mutilation OR "anxiety disord" OR "separation anxiety disord" OR "selective mutism" OR "social anxiety disord" OR "panic disord"” OR agoraphobia OR "generalized anxiety disord"” OR "obsessive compulsive disord"” OR OCD OR "body dysmorphic disord"” OR "hoarding disord"” OR trichotillomania OR excoriation OR "skin-picking disord" OR "trauma disord" OR "stress disord" OR "reactive attachment disord" OR "attachment disord" OR "disinhibited social engagement disord"” OR "post-traumatic stress disord" OR "acute stress disord"” OR "adjustment disord" OR "dissociative disord" OR "dissociative amnesia" OR "depersonalization disord"” OR "derealization disord"” OR "somatic disord"” OR "illness anxiety disord" OR "conversion disord"” OR "feeding disord"” OR "eating disord"” OR pica OR "rumination disord"” OR "avoidant food intake disord" OR "anorexia nervosa" OR anorexia OR "bulimia nervosa" OR bulimia OR "binge eating disord"” OR enuresis OR encopresis OR "sleep disord*" OR insomnia OR "hypersomnolence disord"” OR narcolepsy OR "sex* disord" OR "gender dysphoria" OR "gender identity disord"” OR "behavior disord" OR "disruptive behavior disord" OR "impulse control disord"” OR "conduct disord"” OR "oppositional defiant disord*" OR pyromania OR kleptomania OR "substance disord" OR "substance related disord"” OR "alcohol disord"” OR "cannabis disord"” OR "hallucinogen disord" OR "opioid disord*" OR "neurocognitive disord" "OR delirium OR "traumatic brain injury" OR "personality disord"” OR "schizo* personality disord" OR "paranoid personality disord" "OR "factitious disord" OR psychopath* OR sociopath* OR "antisocial personality disord"” OR "borderline personality disord"” OR "histrionic personality disord" OR "narcissistic personality disord" OR "avoidant personality disord" OR "dependent personality disord"” OR "obsessive compulsive personality disord")

\section{(Deaf OR deaf* OR}

"hard of hearing"

OR "deaf or hard of

hearing" OR "deaf

and hard of hearing"

OR DHH OR

"hearing impair"

OR "permanent

childhood hearing

loss" OR PCHL OR

"sign language")
Concept C: child/ adolescent

Within Concept C, terms used will include:

(Child* OR adolesc* OR juvenile* OR youth OR toddler OR pubescent OR infan*)

(




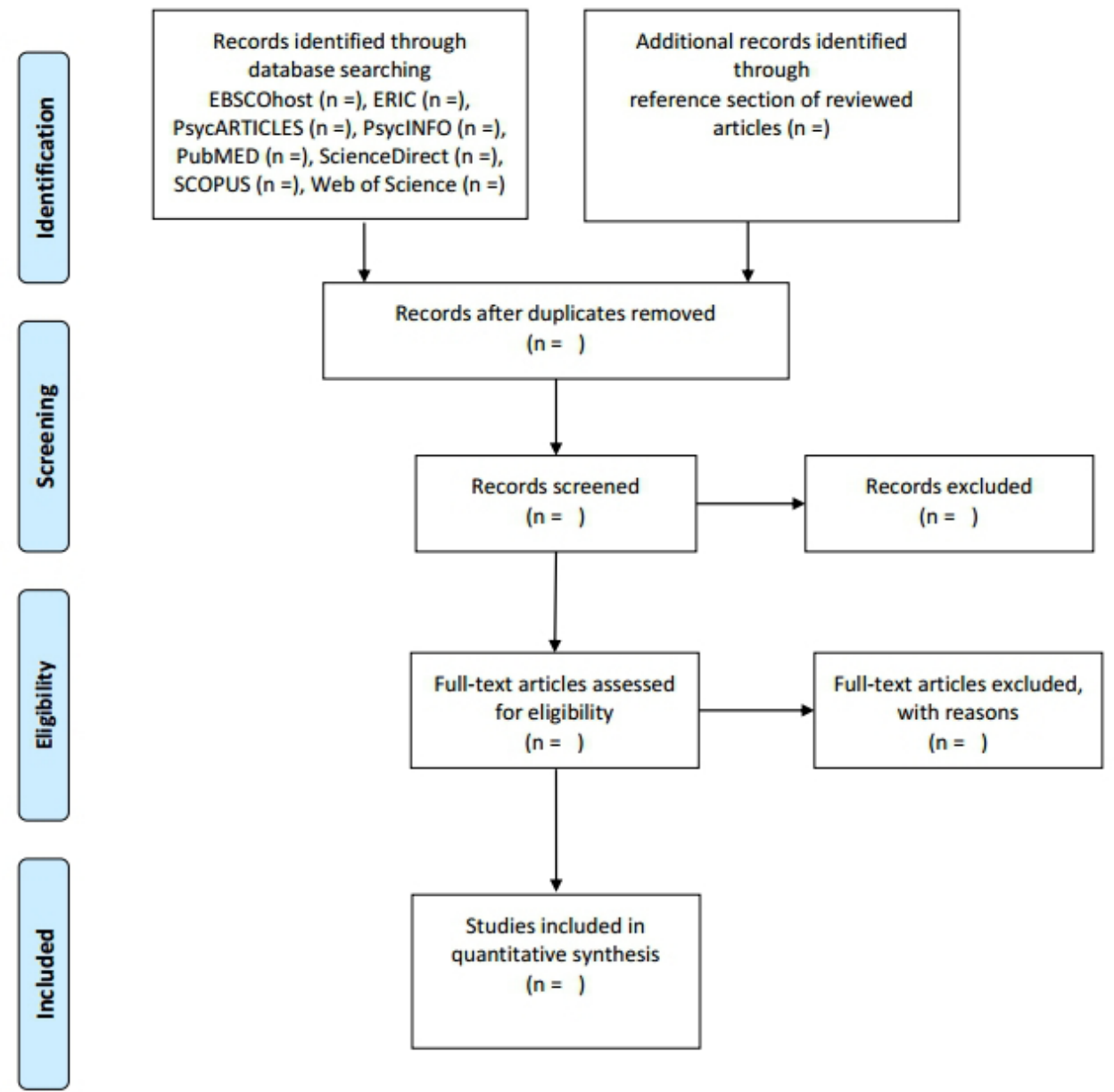

Figure 1 PRISMA 2009 flow diagram. PRISMA, Preferred Reporting Items for Systematic Review and Meta-Analysis.

characteristics, findings and conclusions (see table 3). Extracted data will include study details (author, year of publication, country of study), methodology (study type, inclusion and exclusion criteria, sample size, instruments used to assess disorders and correlates and study participants), sample characteristics (age, sex, coexisting disabilities, type and degree of hearing loss, primary language use, use of cochlear implant or hearing aid, special or mainstream schooling) and findings (types and prevalence rates of disorders and their correlates and confidence intervals).

\section{Quality appraisal and assessment of bias}

We will assess the included studies for quality and risk of bias using the instrument developed by Giannakopoulos et al. ${ }^{24}$ In comparison to other instruments, ${ }^{25-27}$ this instrument was chosen as it is specifically designed to assess quality in prevalence studies that use heterogeneous examination and diagnostic protocols. Moreover, this instrument is validated by an extensive literature review and expert consensus supporting its reliability for use in scientific reviews. Kappa and the interrater correlation coefficient (ICC) were used to test interrater reliability. The latter was assessed on the results of three independent investigators. The ICCs ranged between 0.94 and 1.00 , indicating near perfect agreement between the investigators. The instrument has 11 items that assess sampling, measurement and analysis. It also allows for the calculation of a Total Quality Score (TQS) by totaling the points assigned to each of the items. The TQS ranges from 0 to 4 (poor), 5 to 9 (moderate), 10 to 14 (good) and 15 to 19 (outstanding). TQS scores will not be used to exclude studies but to comment on study quality. Quality appraisal and assessment of bias for each study will be summarised in tabular form and discussed in the review (see table 4).

\section{Data synthesis and analysis}

The study design is quantitative. Extracted data from included studies will be quantified and synthesised to provide a summary of evidence on the prevalence of mental disorders among deaf children and adolescents. A summary of the methodology and results of each included study will also be summarised in tabular form. Finally, the summarised findings will be discussed in a systematic review of existing literature in the field.

\section{Patient and public involvement}

This study involves a review of publicly available published peer-reviewed papers. We did not directly include patient and public involvement in this study. 

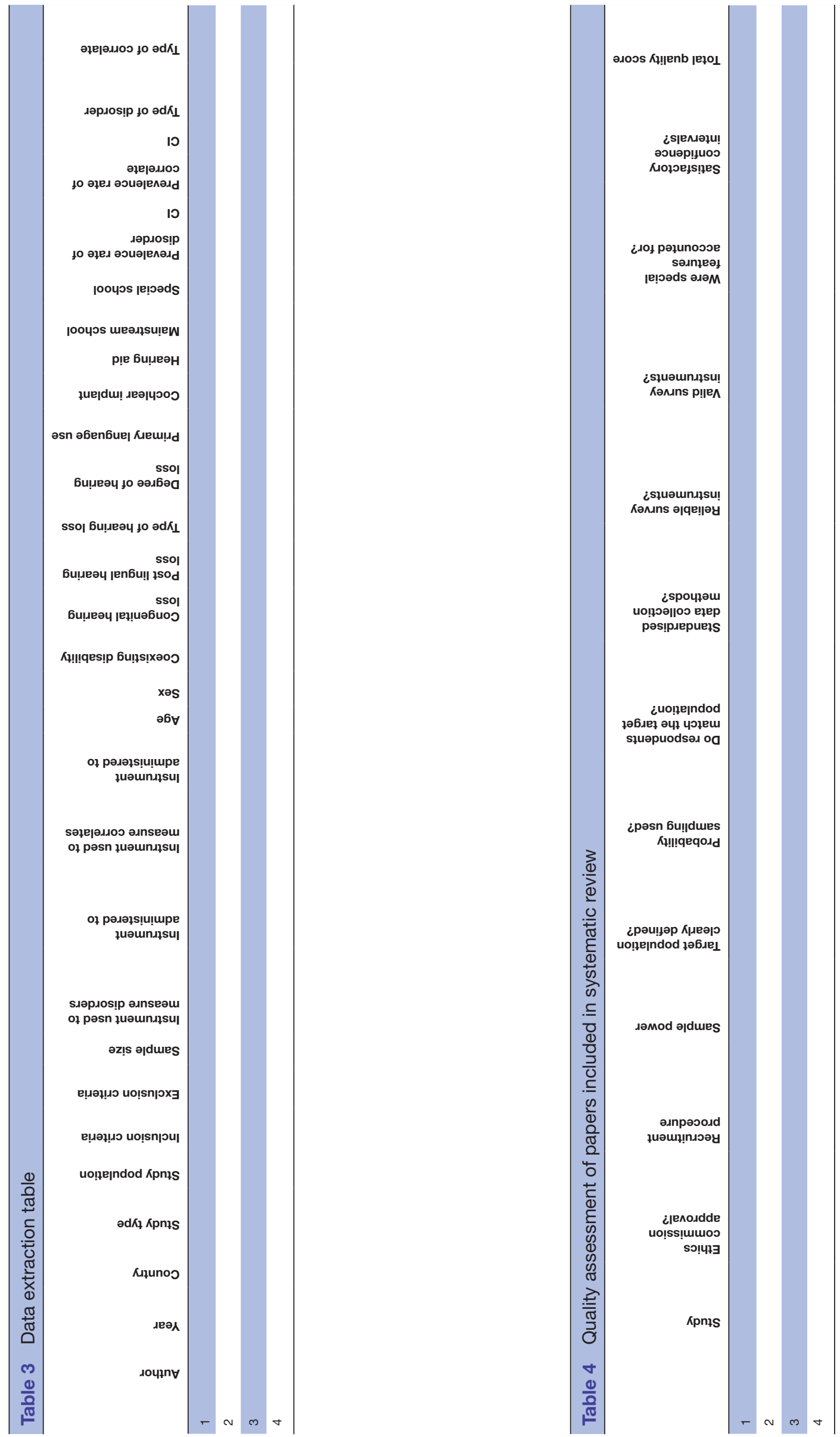


\section{Ethics and dissemination}

This systematic review will use publicly available peerreviewed data from the eight identified search engines (EBSCOHost, ERIC, PsycARTICLES, PsycINFO, PubMED, ScienceDirect, SCOPUS and Web of Science) and will therefore not require an ethical review but an ethics waiver. The systematic review protocol was submitted for ethics waiver clearance with the Stellenbosch University Health Research Ethics Committee as part of a larger study. The findings from this review will be disseminated through peer-reviewed publications.

\section{Author affiliations}

${ }^{1}$ Faculty of Arts and Social Sciences, Department of Psychology, Stellenbosch University, Stellenbosch, Western Cape, South Africa

${ }^{2}$ School of Community and Human Development, Department of Psychology, University of the Witwatersrand, Johannesburg, Gauteng, South Africa

${ }^{3}$ School of Public Health, Division of Epidemiology and Biostatistics, University of the Witwatersrand, Johannesburg, Gauteng, South Africa

Contributors BS, LS and SM contributed to the conception of the study. The protocol was drafted by BS and reviewed by LS, RG and SM. BS and RG will screen all potential studies and extract data from the included studies independently. BS and $R G$ will also assess the risk of bias. BS and RG will conduct data synthesis. LS and SM will arbitrate any review differences and ensure quality assurance during the research process.

Funding The authors have not declared a specific grant for this research from any funding agency in the public, commercial or not-for-profit sectors.

Competing interests None declared.

Patient and public involvement Patients and/or the public were not involved in the design, or conduct, or reporting, or dissemination plans of this research.

Patient consent for publication Not required.

Provenance and peer review Not commissioned; externally peer reviewed.

Open access This is an open access article distributed in accordance with the Creative Commons Attribution Non Commercial (CC BY-NC 4.0) license, which permits others to distribute, remix, adapt, build upon this work non-commercially, and license their derivative works on different terms, provided the original work is properly cited, appropriate credit is given, any changes made indicated, and the use is non-commercial. See: http://creativecommons.org/licenses/by-nc/4.0/.

\section{ORCID iDs}

Brandon Swanepoel http://orcid.org/0000-0002-4956-1461

Leslie Swartz http://orcid.org/0000-0003-1741-5897

Renate Gericke http://orcid.org/0000-0001-9077-1850

\section{REFERENCES}

1 Hindley PA, Hill PD, McGuigan S, et al. Psychiatric disorder in deaf and hearing impaired children and young people: a prevalence study. $J$ Child Psychol Psychiatry 1994;35:917-34.

2 Hintermair M. Prevalence of socioemotional problems in deaf and hard of hearing children in Germany. Am Ann Deaf 2007;152:320-30.

3 Hogan A, Shipley M, Strazdins L, et al. Communication and behavioural disorders among children with hearing loss increases risk of mental health disorders. Aust N Z J Public Health 2011;35:377-83.

4 Vostanis P, Hayes† M, Du FEU M. Behavioural and emotional problems in hearing impaired children: a preliminary study of teacher and parent ratings. Eur J Spec Needs Educ 1997;12:239-46.
5 Remine MD, Brown PM. Comparison of the prevalence of mental health problems in deaf and hearing children and adolescents in Australia. Aust N Z J Psychiatry 2010;44:351-7.

6 Fiorillo CE, Rashidi V, Westgate PM, et al. Assessment of behavioral problems in children with hearing loss. Otol Neurotol 2017;38:1456-62.

7 Van Gent T, Goedhart AW, Hindley PA, et al. Prevalence and correlates of psychopathology in a sample of deaf adolescents. $J$ Child Psychol Psychiatry Allied Discip 2007;48:950-8.

8 Huber M, Kipman U. The mental health of deaf adolescents with cochlear implants compared to their hearing peers. Int $J$ Audiol 2011;50:146-54.

9 Theunissen SCPM, Rieffe C, Kouwenberg M, et al. Behavioral problems in school-aged hearing-impaired children: the influence of sociodemographic, linguistic, and medical factors. Eur Child Adolesc Psychiatry 2014;23:187-96.

10 van Eldik T, Treffers PD, Veerman JW, et al. Mental health problems of deaf Dutch children as indicated by parents' responses to the child behavior checklist. Am Ann Deaf 2004;148:390-5.

11 van Eldik T. Mental health problems of Dutch youth with hearing loss as shown on the youth self report. Am Ann Deaf 2005;150:11-16.

12 Hindley PA. Mental health problems in deaf children. Current Paediatrics 2005;15:114-9.

13 Barker DH, Quittner AL, Fink NE, et al. Predicting behavior problems in deaf and hearing children: the influences of language, attention, and parent-child communication. Dev Psychopathol 2009;21:373-92.

14 Laugen NJ, Jacobsen KH, Rieffe C, et al. Predictors of psychosocial outcomes in hard-of-hearing preschool children. J Deaf Stud Deaf Educ 2016;21:259-67.

15 Fellinger J, Holzinger D, Sattel $\mathrm{H}$, et al. Correlates of mental health disorders among children with hearing impairments. Dev Med Child Neurol 2009;51:635-41.

16 Polat F. Factors affecting psychosocial adjustment of deaf students. J Deaf Stud Deaf Educ 2003;8:325-39.

17 Buskermolen WM, Hoekman J, Aldenkamp AP. The nature and rate of behaviour that challenges in individuals with intellectual disabilities who have hearing impairments/deafness (a longitudinal prospective cohort survey). Br J Learn Disabil 2017;45:32-8.

18 Topol D, Girard N, St Pierre L, et al. The effects of maternal stress and child language ability on behavioral outcomes of children with congenital hearing loss at 18-24months. Early Hum Dev 2011;87:807-11.

19 Black PA, Glickman NS, Demographics GNS. Demographics, psychiatric diagnoses, and other characteristics of North American deaf and hard-of-hearing inpatients. J Deaf Stud Deaf Educ 2006;11:303-21.

20 Sullivan PM, Knutson JF. Maltreatment and behavioral characteristics of youth who are deaf and hard-of-hearing. Sex Disabil 1998;16:295-319.

21 Mejstad L, Heiling K, Svedin CG. Mental health and selfimage among deaf and hard of hearing children. Am Ann Deaf 2009;153:504-15.

22 Bigler D, Burke K, Laureano N, et al. Assessment and treatment of behavioral disorders in children with hearing loss: a systematic review. Otolaryngology-Head and Neck Surgery 2019;160:36-48.

23 Stevenson J, Kreppner J, Pimperton $\mathrm{H}$, et al. Emotional and behavioural difficulties in children and adolescents with hearing impairment: a systematic review and meta-analysis. Eur Child Adolesc Psychiatry 2015;24:477-96.

24 Giannakopoulos NN, Rammelsberg P, Eberhard L, et al. A new instrument for assessing the quality of studies on prevalence. Clin Oral Investig 2012;16:781-8.

25 Munn Z, Moola S, Riitano D, et al. The development of a critical appraisal tool for use in systematic reviews addressing questions of prevalence. Int J Heal Policy Manag 2014.

26 Hoy D, Brooks $P$, Woolf $A$, et al. Assessing risk of bias in prevalence studies: modification of an existing tool and evidence of interrater agreement. J Clin Epidemiol 2012;65:934-9.

27 Loney PL, Chambers LW, Bennett KJ, et al. Critical appraisal of the health research literature: prevalence or incidence of a health problem. Chronic Dis Can 1998;19:170-6. 